\title{
Analysis of a Stochastic Emission Theory Regarding Its Ability to Explain the Effects of Special Relativity
}

\author{
Steffen Kühn \\ Independent Scientist, Berlin, Germany \\ Email: steffen.kuehn@quantino-theory.de
}

How to cite this paper: Kühn, S. (2020) Analysis of a Stochastic Emission Theory Regarding Its Ability to Explain the Effects of Special Relativity. Journal of Electromagnetic Analysis and Applications, 12, 169-187.

https://doi.org/10.4236/jemaa.2020.1212014

Received: November 30, 2020

Accepted: December 28, 2020

Published: December 31, 2020

Copyright $\odot 2020$ by author(s) and Scientific Research Publishing Inc. This work is licensed under the Creative Commons Attribution International License (CC BY 4.0).

http://creativecommons.org/licenses/by/4.0/

\section{cc) (i) Open Access}

\begin{abstract}
In this article, we investigate the physical consequences that would result if electromagnetic field quanta were emitted at random speeds by a source and if the receiver could only perceive the fraction of the quantum field that is slower than the speed of light in its individual rest frame. The analysis shows that this plausible hypothesis eliminates the weak points of conventional emission theories and that both postulates of special relativity are fulfilled. Furthermore, the results demonstrate that this theory can explain numerous experiments that are usually interpreted using different aspects of special relativity. However, the resulting quantum field theory is not equivalent to the special theory of relativity and requires neither spacetime nor Lorentz transformation. Furthermore, this approach offers a starting point for interpreting quantum effects and effects that contradict the special theory of relativity.
\end{abstract}

\section{Keywords}

Emission Theory, Quantized Field, Special Relativity, Temporal

Cross-Section

\section{Introduction}

\subsection{Basic Concept}

Most experiments that analyze aspects of special relativity can be interpreted ambiguously when studied in isolation. However, the special theory of relativity is considered to be the only theory that can explain all experiments with a single approach.

The special theory of relativity is based on two postulates. The first postulate is 
the principle of relativity. With an emission theory ${ }^{1}$, such as that of Walther Ritz [1], this requirement is fulfilled in principle from the outset. However, a conventional emission theory is in direct contradiction with the second postulate, which states:

Measured in any inertial frame of reference, an electromagnetic wave in a vacuum always propagates at speed $c$, independent of the state of motion of the transmitter.

In this article, we investigate whether this requirement can be satisfied with an emission theory.

By assuming that a transmitter emits field quanta according to a probability distribution and that a receiver can only perceive the fraction of the quantum field that is slower or equal to $c$ in its individual rest frame.

In fact, it will become clear that the second postulate of special relativity is fulfilled by this approach. However, now that both postulates are satisfied, a question arises as to whether this emission theory is equivalent to the special theory of relativity. The present article shows that this is not the case, although there are great similarities and similar experimental predictions, even though Lorentz transformation and the spacetime concept can be completely abandoned. Furthermore, we will demonstrate that experiments that have been interpreted as contradictions to the emission theory no longer present a conflict.

A sound introduction to the basic concept of Walther Ritz's emission theory and a discussion of the experimental facts that contradict this theory can be found in [2].

\subsection{Simplified Mathematical Model}

The basic concept described in the previous section shall now be modeled mathematically. To keep the calculations as simple as possible and to provide the reader with some familiarity regarding the mathematics used in this article, some restrictions are made in this section.

Let us assume a point-like transmitter that moves uniformly with velocity $v$ through Newtonian space. Let us further assume that this transmitter emits an electromagnetic pulse at time $t=0$ at the origin of the coordinate system. The trajectory is therefore $\boldsymbol{r}(t)=\boldsymbol{v} t$.

As an emission theory is assumed in this article, we presuppose that there is no medium for propagation of the electromagnetic wave. Instead, it is assumed, as in Ritz's emission theory, that numerous field quanta are constantly emitted from the transmitter and propagate linearly in different directions. Whether these field quanta are photons is of no relevance in the following and will therefore not be further discussed in this article. Furthermore, it is assumed that these field quanta are point-like.

For this article, it is important to note that the field quanta do not move at the speed of light $c$, as is typical in emission theories; rather, the field quanta have

${ }^{1}$ Alternative terms include corpuscular theory, emitter theory, or ballistic theory of light. 
randomly distributed emission speeds $w$. The corresponding probability distribution is hereafter referred to as $\Gamma(w)$.

Because the field quanta propagate at various speeds, we must perform calculations using densities. The quantum density $p(\boldsymbol{r}, t)$ at location $\boldsymbol{r}$ at time $t>0$ can be defined as

$$
p(\boldsymbol{r}, t):=\int_{0}^{\infty} \Gamma(w) \frac{\delta(\|\boldsymbol{r}-\boldsymbol{v} t\|-w t)}{4 \pi(w t)^{2}} \mathrm{~d} w .
$$

We can confirm that this formula (1) is reasonable by inserting $\Gamma(w)=\delta(c-w)$. In this specific case, the formula matches that of a conventional emission theory, and we obtain

$$
p(\boldsymbol{r}, t)=\frac{\delta(\|\boldsymbol{r}-\boldsymbol{v} t\|-c t)}{4 \pi\|\boldsymbol{r}-\boldsymbol{v} t\|^{2}} .
$$

As one can easily see, the term below the fraction line serves to normalize the density. The term above the fraction line, however, determines where the density is different from zero, namely for

$$
\|\boldsymbol{r}-\boldsymbol{v} t\|=c t
$$

It is obvious that this equation describes a sphere with radius $c t$ and center $v t$ at time $t$. The time dependence of the center indicates that, for a normal emission theory, the second postulate of special relativity is violated, as light does not propagate independently of the state of motion of the transmitter.

Now, let us assume that there exists a physical reason that prevents matter from perceiving field quanta moving faster than $c$. A plausible physical reason would be, for example, if matter had a temporal cross-section. Figure 1 illustrates this basic concept and shows that matter may act as a velocimeter at the atomic level.

To mathematically incorporate this effect, the velocity of a field quantum in the rest frame of the receiver must be determined. Such an equation can be easily found, because the ballistic impact speed of a particle such as a field quantum is given by the distance between the transmitter and the receiver at the time of emission divided by the duration required to reach the receiver. For a resting receiver at location $\boldsymbol{r}$, a field quantum emitted at time $t=0$ at the coordinate

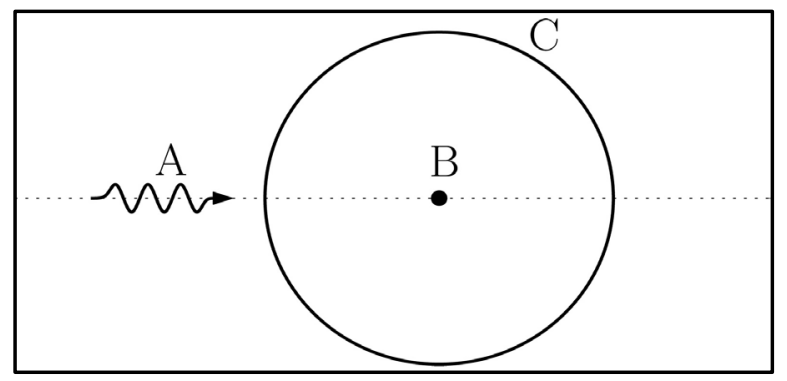

Figure 1. Temporal cross-section: A field quantum (A) can only interact with a particle of matter (B) if it is present in the vicinity $(\mathrm{C})$ of the particle for a sufficient duration. 
origin has the following speed:

$$
u=\frac{\|\boldsymbol{r}\|}{t} .
$$

Consequently, the subjectively perceptible quantum density

$$
\tilde{p}(\boldsymbol{r}, t)=\Theta(c-u) p(\boldsymbol{r}, t)
$$

follows, where $\Theta$ is the Heaviside function. Clearly, the perceptible quantum density is only non-zero if the inequality

$$
\|\boldsymbol{r}\| \leq c t
$$

is fulfilled. Later, we will show that this inequality sign can be replaced by an equal sign.

The inequality (6) leads to the conclusion that an electromagnetic wave cannot be faster than $c$ in the rest frame of a receiver and that this wave has a spherical shape. We can summarize this finding as follows:

A receiver always perceives the propagation of an electromagnetic wave as if the propagation medium were at rest exactly in its individual rest frame.

This statement seems to be paradoxical, and obviously, it is logically impossible for a medium to be simultaneously at rest in all frames of reference. The effect postulated in this article now provides, as one can already guess at this point, a logically satisfying solution for this paradox.

\subsection{Connection to the Lorentz Transformation}

As shown above, the effect studied in this article leads to the conclusion that two receivers moving relative to each other both perceive a resting medium of propagation of the electromagnetic wave in their own frame of reference. Mathematically, this concept can be expressed by two equations:

$$
\|\boldsymbol{r}\|=c t
$$

and

$$
\left\|\boldsymbol{r}^{\prime}\right\|=c t^{\prime},
$$

provided that for $t=t^{\prime}, \quad \boldsymbol{r}=\boldsymbol{r}^{\prime}=\mathbf{0}$ also applies.

Countless articles and textbooks discuss the basic idea of special relativity, which consists in interpreting this seemingly paradoxical feature as an effect of so-called spacetime. The central mathematical construct here is the Lorentz transformation:

$$
\begin{aligned}
& \boldsymbol{r}^{\prime}=\boldsymbol{r}+(\gamma(v)-1) \boldsymbol{r} \cdot \boldsymbol{v} \frac{\boldsymbol{v}}{v^{2}}-\gamma(v) \boldsymbol{v} t \\
& t^{\prime}=\gamma(v)\left(t-\frac{1}{c^{2}} \boldsymbol{r} \cdot \boldsymbol{v}\right),
\end{aligned}
$$

with the Lorentz factor being defined by

$$
\gamma(v):=\frac{1}{\sqrt{1-\frac{v^{2}}{c^{2}}}} .
$$


By inserting the Lorentz transformation (9) into Equation (8), we obtain Equation (7). However, this does not necessarily mean that the Lorentz transformation would be the only or the correct approach for describing nature, if the emission theory postulated in this article is assumed to be true. In the following, we apply an approach that does not employ the spacetime concept. At the same time, the core of a quantum field theory is created, which, due to its scope and far-reaching consequences, must be described and analyzed in separate articles.

\section{Mathematical Model}

\subsection{Quantum Density}

In this section, the initial model of Section 1.2 is generalized to arbitrarily moving transmitters and receivers. Furthermore, we remove the restriction that the electromagnetic pulse is emitted at time $t=0$.

As previously noted, this article investigates an emission theory in which the field quanta are not emitted at a constant speed. Instead, it is assumed that the emission speed $w$ corresponds to a probability distribution $\Gamma(w)$. The specific shape of this distribution is not relevant at this point.

Now, the subjectively perceptible quantum density $\tilde{p}(t)$ shall be calculated in a form that is as general as possible. Let $\boldsymbol{r}_{d}(t)$ be the trajectory of a receiver and $\boldsymbol{r}_{s}(t)$ be the trajectory of a point-like transmitter which permanently emits electromagnetic field quanta. A field quantum that is emitted from the transmitter $\boldsymbol{r}_{s}(\tau)$ at time $\tau$ and moves away with velocity $\boldsymbol{w}$ has the following trajectory:

$$
\boldsymbol{r}_{q}(t, \tau)=\boldsymbol{r}_{s}(\tau)+\left(\boldsymbol{w}+\dot{\boldsymbol{r}}_{s}(\tau)\right)(t-\tau)
$$

for $t>\tau$. If we imagine that at a certain time $\tau$, additional field quanta are emitted in other directions with the same speed $w$, we obtain a sphere whose radius increases with speed $w$. The center of this emission sphere continues to move uniformly according to the following equation:

$$
\boldsymbol{r}_{c}(t, \tau)=\boldsymbol{r}_{s}(\tau)+\dot{\boldsymbol{r}}_{s}(\tau)(t-\tau)
$$

even if the speed of the transmitter changes after the emission. Thus, for the case of an accelerating transmitter, the emission sphere is detached from the transmitter.

Using the emission sphere, it is possible to model the density of the field quanta by the following equation:

$$
p(\boldsymbol{r}, t)=a_{c} \int_{-\infty}^{t} p_{\tau}(\boldsymbol{r}, t, \tau) \mathrm{d} \tau,
$$

with $p_{\tau}$ being defined by

$$
p_{\tau}(\boldsymbol{r}, t, \tau):=\int_{0}^{\infty} \Gamma(w) \frac{\delta\left(\left\|\boldsymbol{r}-\boldsymbol{r}_{c}(t, \tau)\right\|-w(t-\tau)\right)}{4 \pi\left\|\boldsymbol{r}-\boldsymbol{r}_{c}(t, \tau)\right\|^{2}} \mathrm{~d} w .
$$


The parameter $a_{c}$ determines the number of field quanta emitted per second by the transmitter.

Because we have assumed that only field quanta that are not faster than $c$ in the rest frame of the receiver can be perceived, additional filtering is necessary to obtain the subjectively perceivable density $\tilde{p}$. For this purpose, we first consider that a field quantum emitted at time $\tau$ at location $\boldsymbol{r}_{s}(\tau)$ has the following impact velocity:

$$
\boldsymbol{u}(t, \tau)=\frac{\boldsymbol{r}_{d}(t)-\boldsymbol{r}_{s}(\tau)}{t-\tau}-\dot{\boldsymbol{r}}_{d}(t)
$$

at the receiver if it reaches the receiver at time $t$. In this case, $\boldsymbol{r}_{d}(t)$ is the trajectory of the receiver, and $\dot{\boldsymbol{r}}_{d}(t)$ is its velocity at the moment of collision with the field quantum.

Equivalent to the considerations in Section 1.2, this filtering leads to

$$
\tilde{p}(t)=a_{c} \int_{-\infty}^{t} \Theta(c-u(t, \tau)) p_{\tau}\left(\boldsymbol{r}_{d}(t), t, \tau\right) \mathrm{d} \tau
$$

After integrating over $w$, we obtain

$$
\tilde{p}(t)=\frac{a_{c}}{4 \pi} \int_{-\infty}^{t} \frac{\Theta(c-u(t, \tau)) \Gamma\left(\frac{\left\|\boldsymbol{r}_{d}(t)-\boldsymbol{r}_{c}(t, \tau)\right\|}{t-\tau}\right)}{(t-\tau)\left\|\boldsymbol{r}_{d}(t)-\boldsymbol{r}_{c}(t, \tau)\right\|^{2}} \mathrm{~d} \tau .
$$

The subjective quantum density depends only on $t$ and determines the number of field quanta that can be perceived by the receiver in a very small volume element in the vicinity of its own location at time $t$. This equation is valid for arbitrarily moving, accelerating transmitters, as well as for arbitrarily moving, accelerating receivers.

Because the exact form of the $\Gamma(w)$ distribution does not play a major role in this introductory article ${ }^{2}$, it is assumed that

$$
\Gamma(w) \approx \Gamma_{1} w
$$

applies. The fact that the normalization is lost is not important because, due to the postulated effect, a field quantum can only be perceived if its impact speed is lower than $c$. Therefore, the shape of the $\Gamma(w)$ distribution beyond $c+v$ with $|v| \ll c$ is not relevant.

Equation (17) can be simplified via approximation (18) to

$$
\tilde{p}(t)=\frac{a_{c} \Gamma_{1}}{4 \pi} \int_{-\infty}^{t} \frac{\Theta(c-u(t, \tau))}{(t-\tau)^{2}\left\|\boldsymbol{r}_{d}(t)-\boldsymbol{r}_{c}(t, \tau)\right\|} \mathrm{d} \tau .
$$

In the next section, this equation is solved for uniformly moving transmitters and receivers. Based on these results, we can then explore whether and how the emission theory postulated in this paper differs in its experimental predictions

${ }^{2}$ It can be shown that when accounting for the direction of propagation of the field quanta, the magnetic force almost inevitably follows. The shape of the distribution has only a small effect on this result. 
from the special theory of relativity.

\subsection{Solution for Uniformly Moving Transmitters and Receivers}

First, we calculate how an electromagnetic wave emitted by a uniformly moving transmitter is subjectively perceived by a uniformly moving receiver. To keep the calculation as general as possible, the motion is not restricted to the $\mathrm{x}$-axis, as is typically done. Furthermore, light is explicitly considered as a wave.

For this purpose, we add a small oscillation with amplitude $\boldsymbol{d}$ to the trajectory

$$
\boldsymbol{r}_{s}(t)=\boldsymbol{r}_{s 0}+\boldsymbol{v}_{s} t+\boldsymbol{d} \sin (\omega t) \sqcap_{\tau_{0}}^{\tau_{1}}(t)
$$

of the transmitter ${ }^{3}$. This oscillation causes small fluctuations in the quantum density which then propagates in all inertial frames at exactly speed $c$, as will be shown below. To make the calculations even more realistic, this oscillation is additionally switched on at time $\tau_{0}$ using the interval function

$$
\Pi_{a}^{b}(t):=\Theta(t-a)-\Theta(t-b)
$$

and then "switched off" again at time $\tau_{1}$. However, to avoid discontinuities, the two times $\tau_{0}$ and $\tau_{1}$ should be chosen such that the sine term in Equation (20) is zero when the oscillation is switched on and off. This condition is fulfilled when $\tau_{0}$ and $\tau_{1}$ are integer multiples of $\pi / \omega$.

To "measure" the quantum density, a receiver is required. If the receiver is moving uniformly, the trajectory is

$$
\boldsymbol{r}_{d}(t)=\boldsymbol{r}_{d 0}+\boldsymbol{v}_{d} t
$$

with $\boldsymbol{r}_{d 0}$ and $\boldsymbol{v}_{d}$ as freely selectable parameters. By means of the definitions

$$
\boldsymbol{r}_{0}:=\boldsymbol{r}_{d 0}-\boldsymbol{r}_{s 0} \text { and } \boldsymbol{v}:=\boldsymbol{v}_{d}-\boldsymbol{v}_{s},
$$

we get from Equation (15) the approximation

$$
\boldsymbol{u}(t, \tau) \approx \frac{\boldsymbol{r}_{0}+\boldsymbol{v} \tau}{t-\tau}
$$

for very small $\|\boldsymbol{d}\|$.

By inserting (24) into (19), we obtain the subjectively perceivable quantum density for the receiver:

$$
\tilde{p}(t)=\frac{a_{c} \Gamma_{1}}{4 \pi} \int_{-\infty}^{t} \frac{\Theta\left(c-\left\|\frac{\boldsymbol{r}_{0}+\boldsymbol{v} \tau}{t-\tau}\right\|\right)}{(t-\tau)^{2}\left\|\boldsymbol{r}_{d}(t)-\boldsymbol{r}_{c}(t, \tau)\right\|} \mathrm{d} \tau .
$$

The Heaviside function in Equation (25) can be eliminated by considering that this function can only be non-zero if the condition

$$
\left\|\boldsymbol{r}_{0}+\boldsymbol{v} \tau\right\| \leq c(t-\tau)
$$

${ }^{3}$ Electric charge. 
is fulfilled. By squaring both sides, we obtain the following inequation:

$$
r_{0}^{2}+2 \boldsymbol{r}_{0} \boldsymbol{v} \tau+v^{2} \tau^{2} \leq c^{2}(t-\tau)^{2} .
$$

Thus, the condition $\tau \leq \tau_{c}$ with

$$
\tau_{c}:=\frac{c^{2} t+\boldsymbol{r}_{0} \boldsymbol{v}-\sqrt{\left(\boldsymbol{r}_{0} \boldsymbol{v}\right)^{2}+c^{2}\left(\boldsymbol{r}_{0}+\boldsymbol{v} t\right)^{2}-r_{0}^{2} v^{2}}}{c^{2}-v^{2}}
$$

must be fulfilled. We can now simplify Equation (25), and we obtain

$$
\tilde{p}(t)=\frac{a_{c} \Gamma_{1}}{4 \pi} \int_{-\infty}^{\tau_{c}} \frac{(t-\tau)^{-2}}{\left\|\boldsymbol{r}_{d}(t)-\boldsymbol{r}_{c}(t, \tau)\right\|} \mathrm{d} \tau .
$$

In the next step, we insert Equation (12) and the trajectories (20) and (22) and obtain

$$
\tilde{p}(t)=\frac{a_{c} \Gamma_{1}}{4 \pi} \int_{-\infty}^{\tau_{c}} \frac{(t-\tau)^{-2}}{\left\|\boldsymbol{r}_{0}+\boldsymbol{v} t-\boldsymbol{d} k_{s} \Pi_{\tau_{0}}^{\tau_{1}}(\tau)\right\|} \mathrm{d} \tau,
$$

whereas

$$
k_{s}:=(t-\tau) \omega \cos (\omega \tau)+\sin (\omega \tau)
$$

was introduced for reasons of convenience.

To solve this integral, we can exploit the fact that the amplitude $\|d\|$ is a very small quantity. For this case,

$$
\frac{1}{\left\|\boldsymbol{r}_{0}+\boldsymbol{v} t-\boldsymbol{d} k_{s} \Theta(\tau)\right\|} \approx \frac{1}{\left\|\boldsymbol{r}_{0}+\boldsymbol{v} t\right\|}+\boldsymbol{d} \frac{\boldsymbol{r}_{0}+\boldsymbol{v} t}{\left\|\boldsymbol{r}_{0}+\boldsymbol{v} t\right\|^{3}} k_{s} \sqcap_{\tau_{0}}^{\tau_{1}}(\tau) .
$$

Inserting this expression into Equation (30) yields

$$
\tilde{p}(t)=\frac{a_{c} \Gamma_{1}}{4 \pi} \frac{1}{\left\|\boldsymbol{r}_{0}+\boldsymbol{v} t\right\|} \int_{-\infty}^{\tau_{c}} \frac{1}{(t-\tau)^{2}} \mathrm{~d} \tau+\frac{a_{c} \Gamma_{1}}{4 \pi} \boldsymbol{d} \frac{\boldsymbol{r}_{0}+\boldsymbol{v} t}{\left\|\boldsymbol{r}_{0}+\boldsymbol{v} t\right\|^{3}} \int_{-\infty}^{\tau_{c}} \frac{k_{s} \Pi_{\tau_{0}}^{\tau_{1}}(\tau)}{(t-\tau)^{2}} \mathrm{~d} \tau .
$$

Both integrals can be solved. In particular, we can obtain

$$
\int_{-\infty}^{\tau_{c}} \frac{k_{s} \sqcap_{\tau_{0}}^{\tau_{1}}(\tau)}{(t-\tau)^{2}} \mathrm{~d} \tau=\frac{\sin \left(\omega \tau_{c}\right)}{t-\tau_{c}} \Pi_{\tau_{0}}^{\tau_{1}}\left(\tau_{c}\right) \text {. }
$$

This result leads to the quantum density

$$
\tilde{p}=\frac{a_{c} \Gamma_{1}}{4 \pi\left(t-\tau_{c}\right)}\left(\frac{1}{\left\|\boldsymbol{r}_{0}+\boldsymbol{v} t\right\|}+\boldsymbol{d} \frac{\boldsymbol{r}_{0}+\boldsymbol{v} t}{\left\|\boldsymbol{r}_{0}+\boldsymbol{v} t\right\|^{3}} \sin \left(\omega \tau_{c}\right) \sqcap_{\tau_{0}}^{\tau_{1}}\left(\tau_{c}\right)\right),
$$

which describes the number of field quanta that can be perceived by the receiver in a very small volume element in the vicinity of its own location at time $t$. The perceptible ballistic velocity field is obtained by inserting Equation (28) into Equation (24):

$$
\tilde{\boldsymbol{u}}:=\frac{\boldsymbol{r}_{0}+\boldsymbol{v} \tau_{c}}{t-\tau_{c}} .
$$

In the following section, the density $\tilde{p}$ and the velocity field $\tilde{\boldsymbol{u}}$ are interpreted. 


\section{Interpretation}

\subsection{Relativity}

First, one can conclude from Equations (24), (28), (35), and (36) that the principle of relativity is fulfilled, as only the distance $r_{0}$ between the transmitter and receiver at time $t=0$ and the differential velocity $v$ are involved. If the two trajectories of the transmitter and receiver are shifted in space, the equations do not change. Moreover, no changes arise if we add a constant velocity to the transmitter and receiver. Thus, it is clear that any experiment assessing the principle of relativity will not find a violation of the first postulate. Among such experiments, the best known is certainly the Michelson-Morley experiment [3]. Further are the Kennedy-Thorndike experiment [4], the Hammar experiment [5], the Trouton-Noble experiment [6], and the Trouton-Rankine experiment [7].

\subsection{Constancy of the Speed of Light}

The formulas presented above show that electromagnetic waves in all inertial frames always move with speed $c$. To clarify this point, one can, for example, restrict the analysis to the $\mathrm{X}$-axis by setting $\boldsymbol{r}_{0}=r_{0} \boldsymbol{e}_{x}$ and $\boldsymbol{v}=\boldsymbol{v} \boldsymbol{e}_{x}$. In this case, Equation (28) becomes

$$
\tau_{c}=\frac{c t-r_{0}}{c+v}
$$

and for the sine term in Equation (35), we obtain

$$
\sin \left(\omega \tau_{c}\right)=\sin \left(\frac{c}{c+v} \omega\left(t-\frac{r_{0}}{c}\right)\right) .
$$

This means that the oscillation in the quantum density propagates as a running wave with a speed of $c$. In this context, it is quite valid to interpret $r_{0}$ as a position coordinate in the receiver's rest frame, as varying the distance of the receiver at time $t=0$ alters only the value for $r_{0}$ in Equation (38). Two receivers at rest relative to each other thus perceive waves in the same way, only at different positions.

Additionally, the restriction to the $\mathrm{x}$-axis is not necessary. For example, for $v \ll c$, the approximation

$$
\tau_{c} \approx\left(1-\frac{\boldsymbol{r}_{0} \boldsymbol{v}}{r_{0} c}\right)\left(t-\frac{r_{0}}{c}\right)
$$

applies. Hence, we obtain

$$
\sin \left(\omega \tau_{c}\right)=\sin \left(\omega\left(1-\frac{\boldsymbol{r}_{0} \boldsymbol{v}}{r_{0} c}\right)\left(t-\frac{r_{0}}{c}\right)\right)
$$

which again is clearly recognizable as a running wave with speed $c$.

The velocity field $\tilde{\boldsymbol{u}}$ also shows that the fastest field quanta always have a velocity of $c$, because the equation $\|\tilde{u}\|=c$ is valid independent of location or time. 


\subsection{Source Independence and Doppler Effect}

Figure 2 shows the amplitude of $\sin \left(\omega \tau_{c}\right)$ at time $t=0$ for different relative velocities $\boldsymbol{v}=\boldsymbol{e}_{x} v$ with $v=0 c, 0.5 c$, and $0.8 c$. Furthermore, the velocity field $\tilde{\boldsymbol{u}}$ of the field quanta is marked by streamlines.

Figure 2 shows several important aspects. The first is the Doppler effect. As shown by Equation (38), we can arbitrarily change the value of $v$ without altering the characteristic of the wave to move with $c$. Thus, the relative speed of a transmitter does not alter the fact that the wave emitted by the transmitter always propagates at the speed of light $c$ between two mutually resting receivers.

The only effect of $v$ arises for the Doppler factor $c /(c+v)$. As one can easily see, this Doppler factor differs from the value that follows from special relativity for purely longitudinal relative motion, because the multiplication by $1 / \gamma(v)$ is missing. Instead, this Doppler factor corresponds to the acoustic Doppler effect for a receiver that is at rest with respect to the propagation medium.

Seemingly paradoxical, however, is that any observer may consider himself to be at rest in relation to the actually nonexistent medium of propagation. In this respect, this Doppler effect differs from the acoustic Doppler effect, since the latter is different for moving sources and moving receivers. This shows that this theory is in line with effects and experiments concerning the source independence of the speed of light. In particular, these experiments include.

- $\quad$ the double-star experiment of de Sitter [8];

- time-of-flight measurements of gamma photons [9];

- investigations of X-ray binary stars [10].

Moreover, the transverse Doppler effect is not contained in Equation (35). To understand this result, it is useful to examine the middle plot of Figure 2, specifically the propagation directions of the field quanta (white streamlines). The field quanta for do not come directly from the source; rather, their path appears to be curved. This effect is referred to aberration. If one were to measure the transverse Doppler effect directly, as in [11], then, because of the aperture, one would detect field quanta that are moving perpendicular not at the location
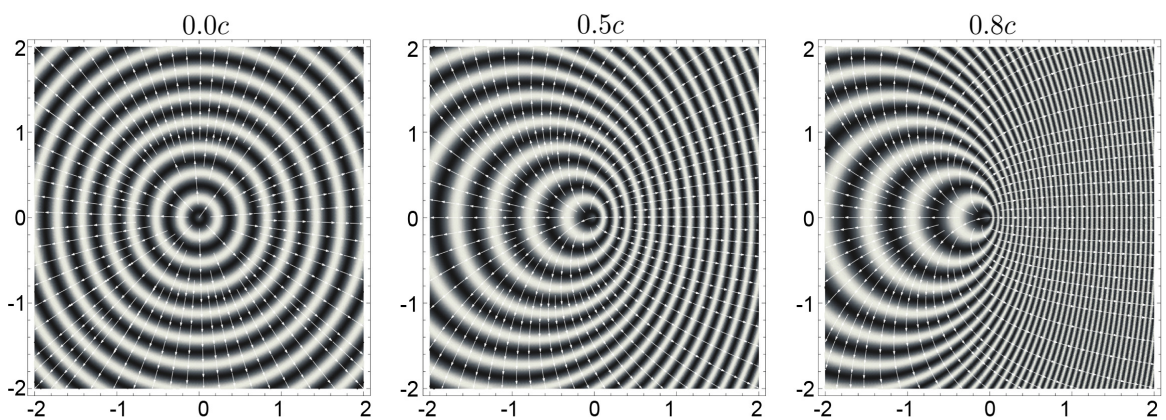

Figure 2. A $30 \mathrm{~cm}$ wave perceived at various relative speeds. The white streamlines show the direction from which the fastest field quanta appear to be approaching. 
$x=0$, but somewhat behind the transmitter. For the fastest field quanta, we can determine that there is neither a red nor a blue shift.

Thus, in Equation (35), the time dilation is missing; otherwise, all aspects of relativity are correctly contained. To account for time dilation, it would be necessary to replace the angular frequency $\omega$ by $\omega / \gamma(v)$, such that the frequency of the source becomes a function of the relative speed of the transmitter and receiver. Arguments that support and oppose this are discussed in Section 3.1.

It is worth noting that the Doppler effect derived here is identical to that given by Klinaku [12]. This correspondence can be recognized by setting the time $t$ in formula (28) to zero, as in Figure 2, and then inserting it into $\sin \left(\omega \tau_{c}\right)$.

\subsection{Coulomb Field}

For completeness, this section will discuss the term that remains when we set $\boldsymbol{d}=\mathbf{0}$ in Equation (35). As previously mentioned, the transmitter of the field quanta is an electric charge. Such a charge can be positive or negative. From this, it follows that there are also positive and negative field quanta. If in Equation (20), a second, inversely oscillating transmitter with opposite sign would have been added, a Hertzian dipole would have been obtained, and the term in question would not exist in Equation (35). This finding shall serve as argumentation to demonstrate that this term is the Coulomb component of the quantum density.

\subsection{No Dispersion in Vacuum}

Here, we analyze the factor $\sqcap_{\tau_{0}}^{\tau_{1}}\left(\tau_{c}\right)$ in Equation (35). This factor arises only because the transmitter in Equation (20) is switched on at time $\tau_{0}$ and then switched off again at time $\tau_{1}$. For $\tau_{c}<\tau_{0}$, the term $\Pi_{\tau_{0}}^{\tau_{1}}\left(\tau_{c}\right)$ equals zero, and the same applies to $\tau_{c}>\tau_{1}$. Therefore, it is important to determine at which times $t$ the condition $\tau_{0} \leq \tau_{c} \leq \tau_{1}$ is valid, as this is the time interval at which the receiver can perceive the oscillation of the source.

By rearranging and solving Equation (28) for $t$, we obtain the following relation:

$$
t=\tau_{c}+\frac{\left\|\boldsymbol{r}_{0}+\boldsymbol{v} \tau_{c}\right\|}{c},
$$

after a skipped calculation. This finding indicates that the activation of the transmitter can only be detected by the receiver when a time span $\left\|\boldsymbol{r}_{0}+\boldsymbol{v} \tau_{0}\right\| / c$ has elapsed. It is noted that $\left\|\boldsymbol{r}_{0}+\boldsymbol{v} \tau_{0}\right\|$ is the distance between the transmitter and receiver at the time when the oscillation is switched on. In contrast, at time $\tau_{1}+\left\|\boldsymbol{r}_{0}+\boldsymbol{v} \tau_{1}\right\| / c$, the receiver perceives that the oscillation disappears. Here again, $\left\|\boldsymbol{r}_{0}+\boldsymbol{v} \tau_{1}\right\|$ is the distance between the transmitter and receiver at exactly the time when the oscillation is turned off.

Thus, a disturbance in the quantum field always propagates spherically with velocity $c$, exactly as if there were a resting propagation medium in the rest frame of the receiver. A particularly important finding, however, is that the elec- 
tromagnetic pulse shows no dispersion ${ }^{4}$, as one might have expected since the field quanta are emitted by the transmitter at random speeds. Electromagnetic waves therefore seem to move in empty space at exactly the speed $c$ and are neither slower nor faster here. Figure 3 shows an example.

\subsection{Aberration}

Another effect linked with special relativity is the aberration of light [13], which is of great importance in astronomy. In cases of aberration, the observation angle $\tilde{\theta}$ of a star does not correspond to the angle of its true position $\theta$, because light has a transit time and because the earth and the star have relative velocities with respect to each other.

This effect is now analyzed with regard to the emission theory considered in this article. Figure 4 shows a transmitter $S$ and a moving receiver $D$, which have a relative velocity $v$ with respect to each other. The gray-shaded field is the velocity field $\tilde{\boldsymbol{u}}$ of the field quanta as it would be perceived from the receiver $D$.

As one can see in the figure, we have

$$
\cos (\theta)=-\frac{r_{0} v}{r_{0} v}
$$

and

$$
\cos (\tilde{\theta})=-\frac{\tilde{\boldsymbol{u}} \boldsymbol{v}}{\tilde{u} v}
$$
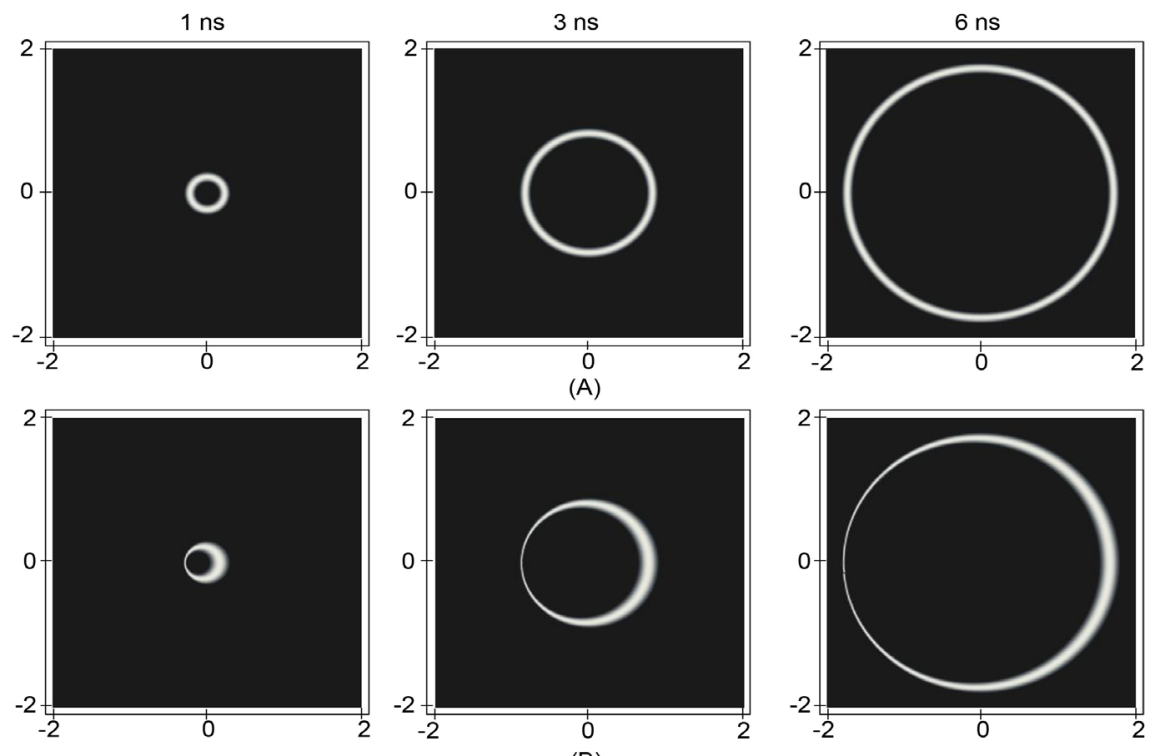

(B)

Figure 3. The propagation of an electromagnetic pulse shown after $1 \mathrm{~ns}, 3 \mathrm{~ns}$ and $6 \mathrm{~ns}$ after its generation. Row (A): in the rest frame of the transmitter, Row (B): in the rest frame of the receiver. The relative speed is $0.8 c$ in $\mathrm{x}$-direction. The plotted function is $\sin \left(\omega \tau_{c}\right) \sqcap_{0}^{\pi / \omega}\left(\tau_{c}\right)$ with $\omega=2 \pi / \lambda$ and $\lambda=30 \mathrm{~cm}$.

${ }^{4}$ Almost, because blueshift or redshift occurs. 


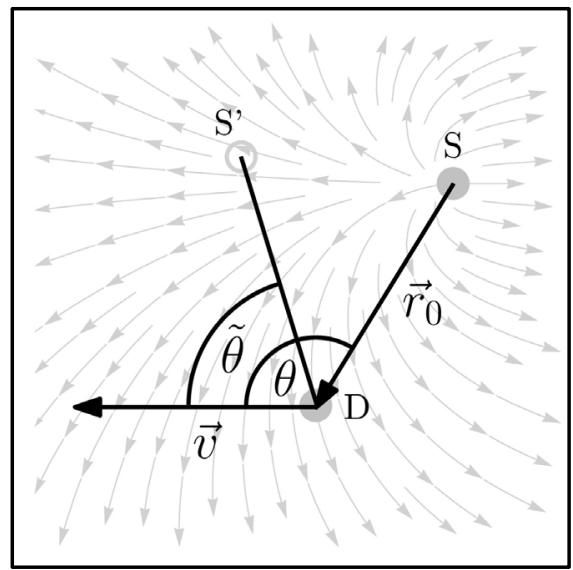

Figure 4. For a moving receiver, the field quanta do not seem to propagate in straight lines, as field quanta that are too fast cannot be perceived due to the temporal cross-section of the receiver. The resulting velocity field is shown in gray. The subjectively perceivable angle of incidence $\tilde{\theta}$ for high relative velocities is clearly different (here, $v=0.8 c$ ) from the true angle $\theta$ between the source and the receiver.

By inserting the velocity field (36) in Equation (43), we obtain

$$
\cos (\tilde{\theta})=-\frac{\boldsymbol{v}}{v} \frac{\boldsymbol{r}_{0}+\boldsymbol{v} \tau_{c}}{\left\|\boldsymbol{r}_{0}+\boldsymbol{v} \tau_{c}\right\|}
$$

By using Equation (28) and Relation (42), we find

$$
\cos (\tilde{\theta})=\frac{v}{c} \sin (\theta)^{2}+\cos (\theta) \sqrt{1-\frac{v^{2}}{c^{2}} \sin (\theta)^{2}}
$$

after a skipped calculation. In the special theory of relativity, however,

$$
\cos \left(\tilde{\theta}_{r e l}\right)=\frac{\frac{v}{c}+\cos (\theta)}{1+\frac{v}{c} \cos (\theta)}
$$

applies [13], which is different from Equation (45). Yet, for $v \ll c$, both equations are identical, and we have

$$
\cos (\tilde{\theta})=\cos \left(\tilde{\theta}_{r e l}\right)=\cos (\theta)+\frac{v}{c} \sin (\theta)^{2}
$$

\subsection{Sagnac Effect}

To analyze this important effect, the transmitter and receiver are positioned on a circle of radius $r$ rotating at angular velocity $\alpha$. The trajectory of the transmit- 
ter is

$$
\boldsymbol{r}_{s}(t)=r\left(\boldsymbol{e}_{x} \sin (\alpha t)+\boldsymbol{e}_{y} \cos (\alpha t)\right),
$$

and the trajectory of the receiver, for instance, a mirror, is

$$
\boldsymbol{r}_{d}(t)=r\left(\boldsymbol{e}_{x} \sin (\alpha t+\psi)+\boldsymbol{e}_{y} \cos (\alpha t+\psi)\right),
$$

with $\psi$ being a fixed angle between the transmitter and receiver. If we insert these expressions into Equation (15), we obtain the ballistic impact velocity of a field quantum emitted by the transmitter at time $\tau$ and arriving at the receiver at time $t$. We find

$$
\boldsymbol{u}(t, \tau)=u_{x} \boldsymbol{e}_{x}+u_{y} \boldsymbol{e}_{y}
$$

with

$$
u_{x}:=r \frac{\sin (\alpha t+\psi)-\sin (\alpha \tau)}{t-\tau}-r \alpha \cos (\alpha t+\psi)
$$

and

$$
u_{y}:=r \frac{\sin (\alpha t+\psi)-\sin (\alpha \tau)}{t-\tau}-r \alpha \cos (\alpha t+\psi)
$$

In the next step, we calculate the Euclidean norm $\|\boldsymbol{u}(t, \tau)\|$ and subsequently the Taylor series. For a small angular velocity $\alpha$, we obtain the following approximation:

$$
\|\boldsymbol{u}(t, \tau)\| \approx r \frac{\sqrt{2(1-\cos (\psi))}}{t-\tau} .
$$

According to the postulate of this article, impact velocities faster than the speed of light cannot be perceived by a receiver. For this reason, the inequality $\|\boldsymbol{u}(t, \tau)\| \leq c$ must be solved again. From this expression, it follows that all $\tau$ values must be less than

$$
\tau_{c}=t-\frac{2 r}{c} \sin \left(\frac{\psi}{2}\right)
$$

Now, we can calculate the speed with which the light pulse propagates. On one hand, this is the velocity

$$
c_{t}:=\frac{\left\|\boldsymbol{r}_{d}(t)-\boldsymbol{r}_{s}\left(\tau_{c}\right)\right\|}{t-\tau_{c}}=c+r \alpha \cos \left(\frac{\psi}{2}\right),
$$

which tells us how fast the light actually moves. In the case of four mirrors arranged in a square, we have $\psi=\pi / 2$ and $c_{t}=c+1 / \sqrt{2} \alpha r$. Because the square has an edge length of $l=\sqrt{2} r$, we finally obtain $c_{t}=c+1 / 2 r \alpha l$.

On the other hand, in the rest frame of the mirrors,

$$
c_{r}:=\frac{\left\|\boldsymbol{r}_{d}(t)-\boldsymbol{r}_{s}(t)\right\|}{t-\tau_{c}}=c
$$

applies, i.e., a measuring device moved along with the mirrors would not detect any deviation from the rule that light in a vacuum moves at speed $c$. 
Therefore, the phase $\phi_{+}$, which results from the passage of light with wavelength $\lambda$ in a rotational direction, is

$$
\phi_{+}=\frac{2 \pi c}{\lambda} \frac{4 l}{c+\frac{1}{2} r \alpha l} .
$$

In the opposite direction, the sign at $\alpha$ is reversed. Therefore, for a small angular velocity $\alpha$, the phase shift between the two partial beams is

$$
\phi_{-}-\phi_{+} \approx \frac{8 \pi l^{2}}{\lambda c} \alpha .
$$

This result corresponds exactly to the equation provided by Sagnac [14].

\subsection{Rotor Experiments}

In this section, we study rotor experiments. For this purpose, let us assume that there is a stationary transmitter at the coordinate origin, which is orbited by a receiver at a distance $r_{d}$. The trajectory of the transmitter is

$$
\boldsymbol{r}_{s}(t)=\boldsymbol{d} \sin (\omega t) \text {. }
$$

Let us further assume that the amplitude $\|\boldsymbol{d}\|$ of the oscillation is very small compared to $r_{d}$. The trajectory of the receiver is defined by

$$
\boldsymbol{r}_{d}(t)=r_{d}\left(\boldsymbol{e}_{x} \sin (\alpha t)+\boldsymbol{e}_{y} \cos (\alpha t)\right),
$$

where $\alpha$ is the angular velocity. Accordingly, $v:=\alpha r_{d}$ is the tangential speed at a distance $r_{d}$.

By inserting the trajectories into Equation (15), we find

$$
u(t, \tau)=r_{d} \sqrt{\frac{1}{(t-\tau)^{2}}+\alpha^{2}},
$$

if we take advantage of the fact that the amplitude $\|\boldsymbol{d}\|$ is very small. Then, inserting (61) into Equation (19) yields

$$
\tilde{p}(t)=\frac{a_{c} \Gamma_{1}}{4 \pi} \int_{-\infty}^{t} \frac{\Theta\left(c-r_{d} \sqrt{\frac{1}{(t-\tau)^{2}}+\alpha^{2}}\right)}{(t-\tau)^{2}\left\|\boldsymbol{r}_{d}(t)-\boldsymbol{r}_{c}(t, \tau)\right\|} \mathrm{d} \tau .
$$

The Heaviside function can be eliminated by considering that this function is non-zero only for

$$
\tau \leq \tau_{c}:=t-\gamma(v) \frac{r_{d}}{c} .
$$

By applying this result and inserting the trajectories (59) and (60), Equation (62) becomes

$$
\tilde{p}(t)=\frac{a_{c} \Gamma_{1}}{4 \pi} \int_{-\infty}^{\tau_{c}} \frac{(t-\tau)^{-2}}{\left\|\boldsymbol{r}_{d}(t)-\boldsymbol{d} k_{s}\right\|} \mathrm{d} \tau .
$$

Here, the term $k_{s}$ is again defined by Equation (31). For very small ampli- 
tudes $d$,

$$
\tilde{p}(t) \approx \frac{a_{c} \Gamma_{1}}{4 \pi} \int_{-\infty}^{\tau_{c}} \frac{r_{d}^{2}+k_{s} d r_{d}(t)}{r_{d}^{3}(t-\tau)^{2}} \mathrm{~d} \tau
$$

applies.

This integral can now be solved, and we obtain

$$
\tilde{p}(t)=\frac{a_{c} \Gamma_{1}}{4 \pi} \frac{r_{d}^{2}+\boldsymbol{d r}_{d}(t) \sin \left(\omega \tau_{c}\right)}{r_{d}^{3}\left(t-\tau_{c}\right)} .
$$

By inserting (63), it follows that

$$
\sin \left(\omega \tau_{c}\right)=\sin \left(\omega\left(t-\gamma(v) \frac{r_{d}}{c}\right)\right),
$$

indicating that there is no transverse Doppler effect.

This result implies that the frequency of the wave does not change. However, as the tangential speed $V$ increases, the speed with which an electromagnetic wave propagates from the transmitter to the receiver decreases. Furthermore, the wavelength appears to be shortened for the receiver, namely, by a factor of $1 / \gamma(v)$. This is consistent with experimental findings, since the often cited experiment by Kündig [15] does not measure the frequency but instead detects an energy change. And a reduction of the wavelength implies a change of the photon energy [16].

\subsection{Time Dilation}

It has become clear that the emission theory discussed in this paper does not provide any time dilation, although the two postulates of special relativity are fulfilled. Therefore, the question arises as to whether time dilation is a real effect or based on a misinterpretation. If the effect is real, it would, in principle, be easy to include it mathematically, as only the frequency of the transmitter is affected and everything else can remain unchanged. Yet this would be illogical. In fact, to the same degree, it is also illogical in the special theory of relativity. The question here is why should time run slower in a completely self-contained system, just because an uninvolved observer is present?

That time dilation due to relative velocities is a very delicate concept is also made clear by thought experiments, such as this one: Two resting clocks are accelerated to speeds $-v$ and $v$. After a certain time span, the clocks are slowed down and accelerated in the opposite direction until they reach speeds $v$ and $-v$. Finally, the clocks are decelerated and come again to rest at the coordinate origin.

The situation is completely symmetrical. If special relativity were correct, each clock would count fewer ticks than the other. This outcome is only possible if one uses the many-worlds interpretation, as proposed by Hugh Everett for quantum mechanics. As this example makes clear, time dilation caused by differential speeds is a very radical assumption, which can by no means be derived 
from the postulates of special relativity, as demonstrated by the existence of this emission theory.

For this reason, time dilation caused by relative velocities should be accepted as real only if there is no other possible explanation at all. However, there are simpler explanations. If one analyzes experiments such as the Hasselkamp experiment [11] or the Ives-Stilwell experiment [17], one notices that ions are initially accelerated and then hit particles at rest. Only at this collision is light generated, which is then analyzed experimentally. As one can easily see, the velocity of the ions is applied twice in very different ways, namely,

1) as the relative velocity between the ion and the measuring device and

2) as the ballistic impact velocity of the ion with respect to the target.

Yet, how can it be ruled out that the frequency drop does not depend on the impact speed and the associated accelerations? As a conclusion, in all experiments that investigate this phenomenon, extreme care must be taken to ensure that the velocities in question only affect the experiment as relative velocities.

\subsection{Further Remarks}

In recent years, researchers have reported an increasing number of indications that it is possible to transfer information at speeds exceeding the speed of light in a vacuum. The ability to transmit information in ordinary copper cables with speeds far beyond $c$ has recently been shown experimentally and theoretically based on the telegrapher's equations [18]. The fact that this ability is predicted by the most fundamental equations of electrical engineering causes much concern. Numerous other experiments have also repeatedly shown that under certain circumstances, it is possible to transmit information at considerable superluminal speeds. The best known experiment is certainly that of Nimtz [19]. The special theory of relativity cannot explain these observations, as each instance of information transmission at superluminal speed is equivalent to a transmission of information into the past [20] [21].

The emission theory postulated here does not have this limitation, as only the speed of the field quanta between two fixed points is limited to a maximum of $c$. Particles of matter, on the other hand, can move relative to each other at any speed. In the context of the emission theory discussed here, the fact that particles cannot be accelerated to speeds faster than $c$ with particle accelerators can be explained by the fact that, at very high relative velocities, the electrical force is reduced to zero in the longitudinal direction of motion and, in turn, increases dramatically in the transverse direction. This fact also results in a nonlinear increase in energy between two systems moving at high relative velocities with respect to each other. However, this finding and the dynamics resulting from this emission theory represent a subject that exceeds the scope of this article.

At this point, we simply mention that the emission theory described herein literally demands the existence of the Lorentz force and enables both a mathematical description and a logical interpretation of this force. For this purpose, it 
is only necessary to combine the quantum density and velocity field into a flux density.

\section{Summary and Conclusion}

The present article has shown that it is feasible that no spacetime exists and that the relativistic effects could be a consequence of the temporal cross-sections of elementary particles. To make this interpretation plausible, we first derived the quantum density of an arbitrarily moving point-like transmitter from the perspective of an arbitrarily moving point-like receiver. Based on this derivation, the field of a uniformly moving, weakly oscillating transmitter was calculated. With this specific solution, we showed that density waves occur in the quantum field, which propagate in all rest frames exactly as if the propagation medium were at rest in all frames simultaneously. Furthermore, we demonstrated that both the principle of relativity and the universal constancy of the speed of light in a vacuum are obtained.

Additionally, it was shown that no dispersion occurs in a vacuum and that there is no dependency of the speed of light on the speed of the source. Thus, the usual objections against emission theories are no longer valid, and it is possible, for example, to explain the Sagnac effect. Furthermore, it became apparent that there is no time dilation caused by relative velocities in the studied emission theory. Although this fact avoids paradoxes, it also leads to the need to interpret certain experiments, such as the Ives-Stilwell experiment, differently, although this seems to be possible. Furthermore, it was shown that the Kündig experiment agrees with theoretical predictions.

Not yet shown in this introductory article is that if the direction of motion of the field quanta is considered, the magnetic force can be derived in a very intuitive way. Furthermore, the wave-particle duality was not discussed, although the reader may have realized that, in the analyzed emission theory, the wave properties of light harmonize well with the particle aspects and that it is easy to interpret the photoelectric effect, for example. Finally, it must be emphasized that the emission theory presented and described herein is intuitive and logical. It not only provides accurate predictions for numerous experiments of special relativity, but also avoids their inherently illogical aspects.

\section{Conflicts of Interest}

The author declares no conflicts of interest regarding the publication of this paper.

\section{References}

[1] Ritz, W. (1908) Recherches critiques sur l'Électrodynamique générale. Annales de Chimie et de Physique, 13, 145.

[2] Fox, J.G. (1965) Evidence against Emission Theories. American Journal of Physics, 33, 1-17. https://doi.org/10.1119/1.1971219 
[3] Michelson, A.A. and Morley, E.W. (1887) On the Relative Motion of the Earth and the Luminiferous Ether. American Journal of Science, 34, 333-341. https://doi.org/10.2475/ajs.s3-34.203.333

[4] Kennedy, R.J. and Thorndike, E.M. (1932) Experimental Establishment of the Relativity of Time. Physical Review, 42, 400-418.

https://doi.org/10.1103/PhysRev.42.400

[5] Hammar, G.W. (1935) The Velocity of Light within a Massive Enclosure. Physical Review, 48, 462-463. https://doi.org/10.1103/PhysRev.48.462.2

[6] Trouton, F.T. and Noble, H.R. (1903) The Forces Acting on a Charged Condenser Moving through Space. Proceedings Royal Society London, 74, 132-133.

[7] Trouton, F.T. and Rankine, A. (1908) On the Electrical Resistance of Moving Matter. Proceedings of the Royal Society of London, 80, 420-435.

[8] de Sitter, W. (1913) Ein astronomischer Beweis für die Konstanz der Lichtgeschwindigkeit. Physikalische Zeitschrift, 14, 429.

[9] Alväger, T., Nilsson, A. and Kjellman, J. (1963) A Direct Terrestrial Test of the Second Postulate of Special Relativity. Nature, 197, 1191. https://doi.org/10.1038/1971191a0

[10] Brecher, K. (1977) Is the Speed of Light Independent of the Velocity of the Source? Physical Review Letters, 39, 1051-1054. https://doi.org/10.1103/PhysRevLett.39.1051

[11] Hasselkamp, D., Mondry, E. and Scharmann, A. (1979) Direct Observation of the Transversal Doppler-Shift. Zeitschrift für Physik A Atoms and Nuclei, 289, 151-155. https://doi.org/10.1007/BF01435932

[12] Klinaku, S. (2016) The Doppler Effect and the Three Most Famous Experiments for Special Relativity. Results in Physics, 6, 235-237. https://doi.org/10.1016/j.rinp.2016.04.011

[13] Tatum, J. (2020) Celestial Mechanics. 1em plus 0.5em minus 0.4em LibreTexts.

[14] Sagnac, G. (1913) Léther lumineux démontré par l'effet du vent relatif d'éther dans un interféromètre en rotation uniforme. Comptes Rendus Physique, 95, 708-710.

[15] Kündig, W. (1963) Measurement of the Transverse Doppler Effect in an Accelerated System. Physical Review, 129, 2371. https://doi.org/10.1103/PhysRev.129.2371

[16] Barnett, S.M. and Loudon, R. (2010) The Enigma of Optical Momentum in a Medium. Philosophical Transactions of the Royal Society A, 368, 927. https://doi.org/10.1098/rsta.2009.0207

[17] Ives, H.E. and Stilwell, G. (1938) An Experimental Study of the Rate of a Moving Atomic Clock. Journal of the Optical Society of America, 28, 215-226. https://doi.org/10.1364/JOSA.28.000215

[18] Kühn, S. (2020) General Analytic Solution of the Telegrapher's Equations and the Resulting Consequences for Electrically Short Transmission Lines. Journal of Electromagnetic Analysis and Applications, 12, 71-87.

[19] Nimtz, G. (2011) Tunneling Confronts Special Relativity. Foundations of Physics, 41, 1193-1199. https://doi.org/10.1007/s10701-011-9539-2

[20] Bohm, D. (1965) The Special Theory of Relativity. W.A. Benjamin, New York.

[21] Tolman, R.C. (1917) The Theory of the Relativity of Motion. University of California Press, Berkeley. 